\title{
The perspective matters! Multisensory integration in ego- centric reference frames determines full-body ownership
}

\section{Valeria I. Petkova*†, Mehrnoush Khoshnevis ${ }^{\dagger}$ and H. Henrik Ehrsson}

Brain, Body and Self Laboratory, Department of Neuroscience, Karolinska Institutet, Stockholm, Sweden

\section{Edited by:}

Anna M. Borghi, University of Bologna, Italy

\section{Reviewed by:}

Manos Tsakiris, University of London,

UK

G. Lorimer Moseley, University of

South Australia, Australia

*Correspondence:

Valeria I. Petkova, Department of Neuroscience, Karolinska Institute,

Retzius väg 8, SE-171 77 Stockholm,

Sweden.

e-mail: valeria.petkova@ki.se

${ }^{+}$Valeria I. Petkova and Mehrnoush

Khoshnevis have contributed equally to this work.
Recent advances in experimental science have made it possible to investigate the perceptual processes involved in generating a sense of owning an entire body. This is achieved by full-body ownership illusions which make use of specific patterns of visual and somatic stimuli integration. Here we investigate the fundamental question of the reference frames used in the process of attributing an entire body to the self. We quantified the strength of the body-swap illusion in conditions where the participants were observing this artificial body from the perspective of the first or third person. Consistent results from subjective reports and physiological recordings show that the first person visual perspective is critical for the induction of this full-body ownership illusion. This demonstrates that the multisensory integration processes producing the sense of corporeal self operates in an ego-centric reference frame.

\section{Keywords: body ownership, first person perspective, multisensory integration, perceptual illusion}

\section{INTRODUCTION}

How do we come to experience our body as part of ourselves? Does the mind have the capacity to attribute an entire new body to the self? These questions are fundamental to our understanding of the relationship between the body, the mind, and the sense of self (James, 1890; Gallagher, 2000; Metzinger, 2003; Merleau-Ponty, 2005).

Recent advances in experimental science have made it possible for cognitive neuroscientists to address this important aspect of self-awareness in controlled laboratory settings (Botvinick and Cohen, 1998; Jeannerod, 2003; Ehrsson et al., 2004; Makin et al., 2008; Blanke and Metzinger, 2009; Petkova and Ehrsson, 2010). One particularly powerful approach is the use of "full-body illusions" in which healthy individuals experience a new artificial body as their own (Ehrsson, 2007; Lenggenhager et al., 2007; Petkova and Ehrsson, 2008; Slater et al., 2009). This approach follows a long tradition in psychology and neuroscience of studying perceptual illusions to learn more about the basic processes that underlie normal perception. In one such full-body illusion, the "body-swap illusion" (Petkova and Ehrsson, 2008), the participants are able to sense the touches applied to the body of a shop mannequin and experience this body as their own. This illusion is elicited in the following way: the participants observe the artificial body via head-mounted displays (HMD) connected to video-cameras placed on the mannequin's head. The cameras are positioned so that they look down at the mannequin's body, and thus, the participants see the mannequin's body at the location where they would normally see their own body. The illusion is evoked when the experimenter applies identical synchronous touches to the body of the mannequin and the participant's body. The illusion is abolished, or significantly reduced, if the human body is replaced with an object that does not have a humanoid shape (e.g., a block of wood) or if asynchronous touches are applied to the two bodies (Petkova and
Ehrsson, 2008). This findings suggest at the core of this perceptual phenomenon is the integration of multiple sources of sensory information centered on the spatial location of the "new" body (Petkova and Ehrsson, 2008).

Still unanswered, however, is the important question of the role played by the visual perspective in the process of perceiving an entire body as one's own. In spatial cognition research, a basic distinction is made between the first person perspective (1PP) and the third person perspective (3PP; Vogeley and Fink, 2003), related to ego-centric and allocentric reference frames, respectively (Klatzky, 1998; Burgess, 2006). An ego-centric reference frame is a coordinate system centered on the body, and is considered to be important for functions related to perception and to performing actions (Fogassi et al., 1992; Graziano and Gross, 1998). In contrast, the allocentric reference frame corresponds to world coordinates centered on a reference point in extrapersonal space. This coordinate system is considered important for spatial cognitive functions such as determining one's location with respect to environmental landmarks, spatial navigation, and spatial memory (Maguire et al., 1998; Burgess, 2006).

The uncertainty about the role played by the visual perspective in feeling ownership of a body arises both from experimental results from full-body illusions in healthy individuals (see below) and from certain neurological conditions which suggest that the relation between self-awareness and the first person visual perspective is not straightforward. For example, in evoked or spontaneous cases of autoscopy, a form of out-of-body experience in which people perceive themselves as being outside their own body looking at it from an altered egocentric perspective, people still report that the body they observe is their own, thus, they demonstrate intact body self-attribution, despite the fact that they are viewing the body from a 3PP (Blanke et al., 2002, 2004; Blanke and Mohr, 2005). 
More directly relevant for the question of how a normally functioning mind creates a multisensory experience of one's own body, as is under consideration here, is the fact that two principally different experimental set-ups have been used to induce "full-body illusions" associated with changes in perceived body ownership in healthy participants (Lenggenhager et al., 2007; Petkova and Ehrsson, 2008). In these experiments, synchronous visual and tactile stimulation is always applied both to the artificial body in sight of the participant and the participant's own body which is out of sight. However, the illusory "own" body is either viewed from a 3PP, as though looking at another individual a couple of meters in front of oneself (Lenggenhager et al., 2007, 2009; Aspell et al., 2009), or from a 1PP (Ehrsson, 2007; Petkova and Ehrsson, 2008; Slater et al., 2009, 2010), as though directly looking down at one's body. Logically, if the visual perspective is irrelevant, one would anticipate that the body ownership illusion would be evoked equally well in these two situations. Hence, some authors have concluded that body ownership would depend solely on the detection of visuo-tactile synchrony (Meyer et al., 2008).

Here, we sought to address this important question and to investigate which of the two basic visual perspectives is most important for the perceptual illusion of owning an entire artificial body and, therefore, for the general mechanisms of attributing a body to oneself. In two different behavioral set-ups, we directly compared experimental conditions in which the participants observed a body of a mannequin from either the first or the third person visual perspective. We hypothesized that the first person visual perspective would represent a fundamental constraint on the full-body illusion. This would be in line with the proposed model that the sense of body ownership relies on multisensory integration mechanisms operating in body-part-centered reference frames (Ehrsson et al., 2004; Makin et al., 2008; Petkova and Ehrsson, 2008; Ehrsson, 2011).

\section{MATERIALS AND METHODS PARTICIPANTS}

A total of 50 naive healthy participants were recruited to the study, comprised of three separate behavioral experiments. In the first behavioral experiment, we tested a group of 20 male participants (mean age and SD: $24 \pm 5$ years), in the second 13 male volunteers were included (mean age and SD: $27 \pm 4$ years), and in the third, 17 male participants were recruited (mean age and SD: $24 \pm 4$ years). All participants were male to exclude possible confounding effect of gender as both mannequins used in the experiment were male. The Ethical Review Board of Karolinska Institutet approved the experimental protocol and written informed consent was obtained from each participant.

\section{EXPERIMENTAL PROCEDURES \\ Experiment \#1}

During the experiment, participants wore a set of HMDs (Cybermind Visette Pro PAL, Cybermind Interactive, Maastricht, the Netherlands) with a wide field-of-view (diagonal field-ofview $=71.5^{\circ}$; following the procedures described in Petkova and Ehrsson, 2008). These HMDs were connected to two synchronized color CCTV cameras (Protos IV, Vista, Wokingham, Berkshire, UK) attached side-by-side to a special platform mounted on the head of a full size shop mannequin (mannequin \#1). The platform upon which the CCTV cameras were mounted was fixed on a rotating axis, which allowed the cameras to be pointed either downward to the body of the mannequin (mannequin \#1), or forward toward the body of second identical mannequin (mannequin \#2) placed opposite mannequin \#1 at a distance of $75 \mathrm{~cm}$ from the participant (Figure 1). Thus, the participants could not see their own body but only the body of mannequin \#1 or \#2 from one or other of the two different points of view (see further below).

The experiment consisted of two conditions, each of which lasted for $2 \mathrm{~min}$. The order of the conditions was balanced across the participants. In the condition corresponding to the $1 \mathrm{PP}$, the participant was asked to tilt his head downward as if to look at his own body while actually looking through the HMDs at the body of mannequin \#1 at a location and orientation similar to the participant's own body. In the second condition, referred to as the 3PP condition, the participant was asked to position his head as if to look forward toward the body of a person standing just opposite him. In the HMDs, the participant saw the body of mannequin \#2 facing him at a distance of $75 \mathrm{~cm}$. In both conditions the experimenter used two plastic rods to apply the same number of synchronized strokes on the right lateral parts of the mannequin's abdomen and the participant's abdomen. After each condition, the participants were asked to complete a questionnaire, in which they had to affirm or deny nine possible perceptual effects using a seven-point Likert scale (Figure 3A).

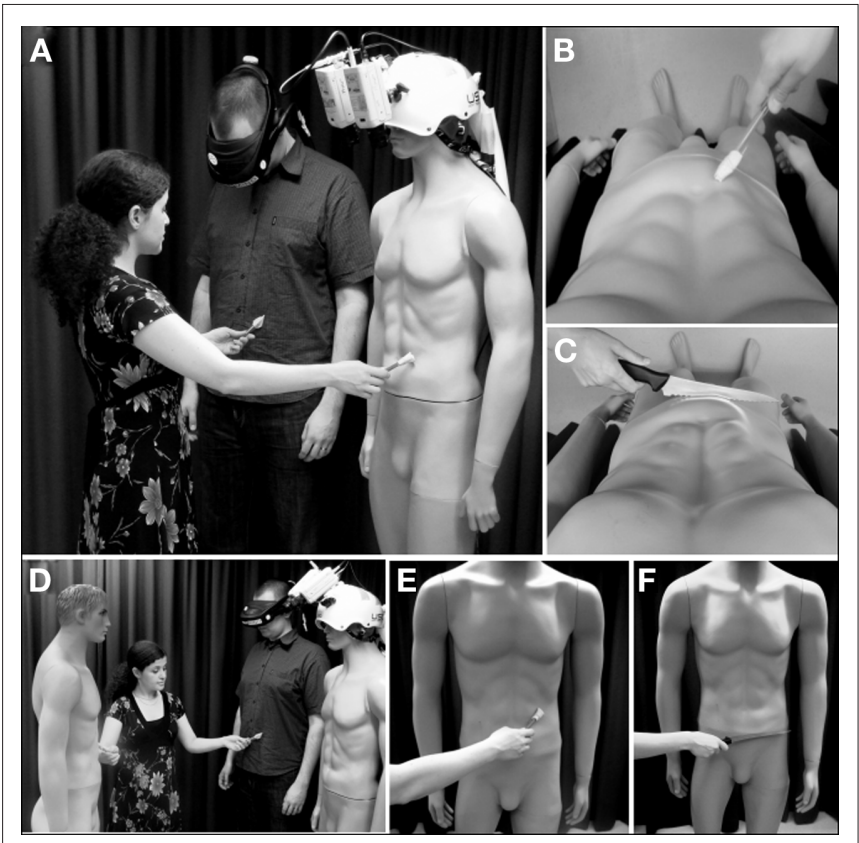

FIGURE 1 | Experimental set-up in experiments \#1 and \#2. (A) Set-up used to create the full-body illusion when the mannequin's body is perceived from the first person perspective. (B,C) Depict what the participants see when they observe the touches or the knife-induced threats in the first person perspective condition. (D) Set-up designed to probe the full-body illusion when the mannequin's body is perceived from the third person perspective. (E,F) Depict the participants' perspective when they received touches or knife threats in the condition presented from the third person perspective. 


\section{Experiment \#2}

The experimental set-up and general procedures were identical to those used in the first experiment, but instead of using a questionnaire to probe for subjective illusion-related experiences, we used a knife to inflict injury on the mannequin, while registering the skin conductance responses (SCR) of the participants. The threat-evoked SCR is related to autonomic nervous system arousal when the own body is physically threatened and can be used as an objective index of the illusion (as described in detail in Petkova and Ehrsson, 2008). The experiment consisted of four conditions, repeated three times in a pseudo-randomized order. In two conditions the body of the mannequin was seen from the 1PP and the timing of the visuo-tactile stimulus was either synchronized (1PP sync) or asynchronized (1PP async). In the two other conditions the body was seen from the 3PP (3PP sync vs. 3PP async). Each condition lasted $1 \mathrm{~min}$ and was immediately followed by a "threat event" during which the experimenter was perceived to cut with a knife the abdomen of the mannequin being looked at. The knife was slid along the mannequin's abdomen, and the motion was performed so that the knife was always moved along the horizontal axis from left to right in the field-of-view of the HMDs (the whole event took approximately $3 \mathrm{~s}$ ). The onsets of these threat events were flagged in the computer files recording the SCRs, and the magnitude of the related conductance changes was calculated according to the procedures described in Petkova and Ehrsson (2008).

\section{Experiment \#3}

Here, we created a new set-up that allowed the participants to experience the illusion of owning the mannequin's body without the use of HMDs and video-cameras. The set-up used for the first person visual perspective conditions was as follows: the participant was asked to lay on a bed tilted at $30^{\circ}$ from the horizontal. The front half of the body of a shop mannequin was positioned on top of the participant so that the shoulders of the mannequin were resting on the shoulders of the participant and the feet of the mannequin were resting on a horizontal support positioned $\sim 50 \mathrm{~cm}$ above the feet of the participant. Thus, when the participant looked down as if to look at his body, he saw the body of the mannequin in a similar position to where he would expect his own body to be had he been to lying down horizontally (Figures 2A-C). The experimenter used two plastic rods to apply touches on the right lateral part of the abdomens of the mannequin and the participant, either in a synchronous manner (1PP Sync) or in an asynchronous mode (1PP Async). While this was going on, the experimenter was out of sight, hidden behind a curtain, to prevent the participants from seeing the experimenter's hand touching the participant's own abdomen. Thus, the only hand the participant saw was the experimenter's hand touching the abdomen of the mannequin. After 1 min of synchronous or asynchronous tactile stimulation, the experimenter used a knife to "cut" the mannequin's abdomen in a single movement lasting approximately $3 \mathrm{~s}$. The SCRs associated with these threat events were registered and analyzed as explained above and in the previously published protocols (Petkova and Ehrsson, 2008).

The set-up used to test the illusion when the mannequin was viewed from a 3PP was as follows: Mannequin \#2 was placed on an identical bed as the one the participant was lying on. This bed was tilted at the same angle (approx $30^{\circ}$ ), but turned in the opposite

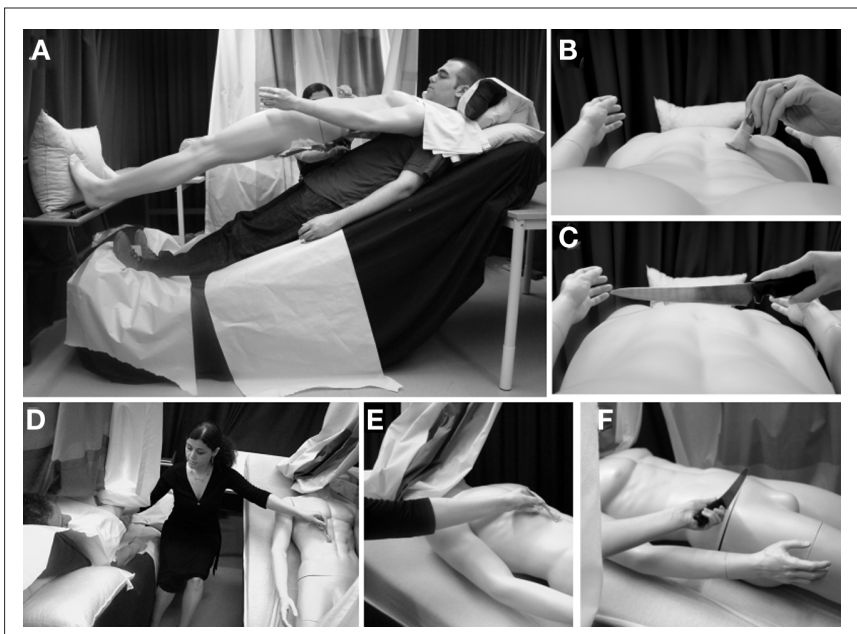

FIGURE 2 | Experimental set-up developed to induce the body-swap illusion without head-mounted displays and video-technology (experiment \#3). (A-C) The experimental set-up and the participant's field of sight in the conditions in which the mannequin's body was viewed directly from the first person perspective. (D-F) The set-up developed to probe the full-body illusion when the mannequin's body was perceived from the third person perspective.

direction $1 \mathrm{~m}$ to the right of the participant's bed (Figures 2D-F). The participant was asked to turn his head to the right and look at the body of mannequin $\# 2$ on the other bed. A curtain was positioned over the body of mannequin \#1 resting on top of the participant's body (see previous paragraph) to hide it from the participant. The curtain was also occluding the experimenter, who applied synchronous (3PP sync) or asynchronous (3PP async) touches on the right lateral part of the abdomens of the participant and mannequin \#2. We used an identical threat-procedure to that when "cutting" the mannequin with a knife, as described above, and registered the associated SCRs. The four conditions (1PP Sync, 1PP Async, 3PP Sync, and 3PP Async) were repeated three times in an order that was balanced over all the participants.

\section{RESULTS \\ EXPERIMENTS 1 AND 2: ELICITATION OF THE ILLUSION USING HEAD- MOUNTED DISPLAYS AND VIDEO-TECHNOLOGY}

We first examined the role of the visual perspective (first vs. third person) for the elicitation of the full-body illusion by using a modified version of our previously published "body-swap illusion" (Petkova and Ehrsson, 2008; see Materials and Methods). In the first experiment, we used a questionnaire to quantify the subjective experience of the two experimental conditions in a group of 20 naive participants. The results showed that 17 out of the 20 participants perceived that the mannequin's body was their own body when viewed from the $1 \mathrm{PP}$. In contrast, only seven out of 20 affirmed this experience when they viewed the body from the third person visual perspective (Figure 3A). Importantly, in the 1PP condition, the mean rating scores for the three illusion questions were greater than for the six control questions, and this difference was greater than in the $3 \mathrm{PP}$ condition. Thus, there were significant differences between the levels of the factors "Perspective" [first vs. 


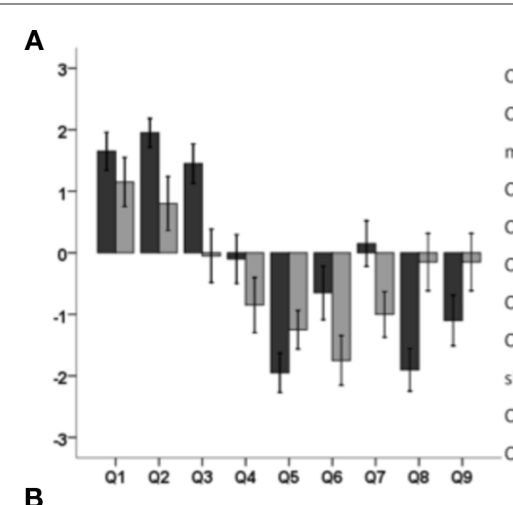

B

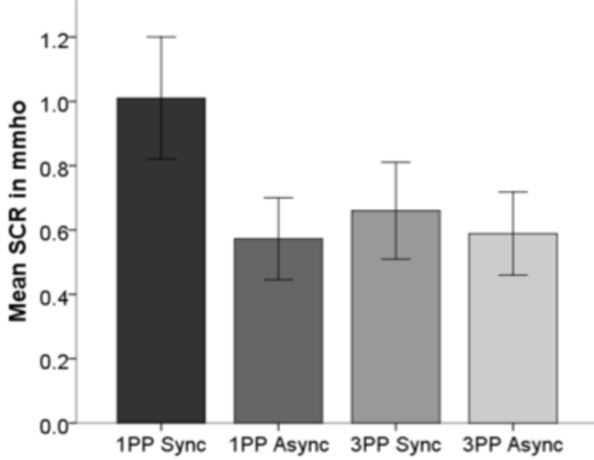

FIGURE 3 | Introspective and objective evidences from the three experiments. (A) Experiment \#1: The participants rated the illusion-related questions significantly higher than the control questions; this difference was significantly greater when the mannequin's body was perceived from the first person perspective (i.e., significant interaction of the main factors "Perspective" vs. "Question type"). Dark bars represent the ratings in the first person perspective and light bars represent the ratings in the third person perspective respectively. The error bars indicate standard errors. (B) Experiment \#2: The
Q1: I seemed to feel the touch given to the mannequin

Q2: It seemed as though the touch I felt was caused by the stick touching the

mannequin's body

Q3: It felt like the mannequin's body was my body

Q4: I felt naked

Q5: I felt like I had two bodies (at the same time)

Q6: It felt as if my body had turned into a plastic body

Q7: The mannequin's body began to resemble my own body in terms of shape,

skin tone, or some other visual features

Q8: I felt like I was looking in a mirror

Q9: It felt as if the body I saw belonged to someone else

C

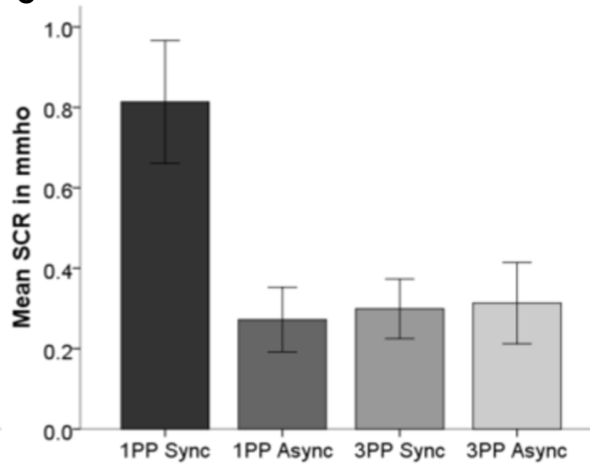

threat-evoked increase in the skin conductance responses (SCR) was significant only in the condition in which the participant observed the synchronized visuo-tactile stimulation from the first person perspective. The error bars indicate standard errors. (C) Experiment \#3: The full-body illusion can be evoked without the help of HMDs and video-technology. The specific threat-evoked increase in the SCR when the body was observed from the first person perspective again emphasizes the importance of this visual perspective for the mechanism of attributing a body to oneself. The error bars indicate standard errors. third; $F(1,19)=5.787, p=0.026$ ], and "Question type" [illusion vs. control; $F(1,19)=111.914, p<0.001$ ] and, crucially, a significant interaction between the two factors $[F(1,19)=10.226, p=0.005]$ in two-way $2 \times 2$ ANOVA.

Objective evidence for this effect was obtained in a second experiment using a different group of 13 naïve participants. Here, we first induced the illusion using the procedures described above and then "physically threatened" the mannequin by moving a knife along the abdomen of the mannequin's body. We then measured the resulting changes of the SCRs. To control for the unspecific effect of seeing a knife, we used a $2 \times 2$ factorial design where we systematically varied the visual perspective and the synchronicity of the applied touches to the mannequin's and the participant's bodies because asynchronous touches on the two bodies have been shown to eliminate or significantly reduce the illusion (Petkova and Ehrsson, 2008). We found that the threat-evoked physiological reaction was significantly greater in the condition with synchronous touches when the mannequin was observed from the 1PP than when it was seen from the 3PP. Statistically, this corresponded to a significant interaction between the main factors "Perspective" (first vs. third) and "Timing of the visual and tactile stimuli" [synchronous vs. asynchronous; $F(1,12)=6.876, p=0.022$, two-way ANOVA]. The main effect of the factor "Perspective" did not reach significance $F(1,12)=2.761, p=0.122$, however the levels of the factor "Timing" were significantly different $F(1,12)=5.160, p=0.042$ (Figure 3B). Direct pairwise comparisons of the synchronous and asynchronous conditions reached significance only when the mannequin was observed from the $1 \mathrm{PP}(p=0.017$; see Figure 1B). Thus, both the physiological and the questionnaire data showed that the first person visual perspective is an important factor for attributing the body of a mannequin to one's self.

\section{EXPERIMENT 3: ELICITING THE ILLUSION WITHOUT USING HEAD- MOUNTED DISPLAYS AND VIDEO-TECHNOLOGY}

Next, we reproduced our findings in a real life set-up and excluded the possibility that the illusion depends on the video-technology used. For this, we devised an experimental set-up in which the body of the mannequin could be observed directly, either from the 1PP or from the 3PP (Figure 2). In a new group of 17 naïve volunteers, we made use of the threat-evoked SCRs to quantify the illusion objectively. As expected, we observed significantly greater threat-evoked responses in the condition when the body was observed from the $1 \mathrm{PP}$ and the touches on the two bodies were synchronized (a significant interaction between the main factors "Perspective" (first vs. third) and "Timing of visual and tactile stimulation" [ synchronous vs. asynchronous; $F(1,16)=4.727, p=0.045$, two-way ANOVA]. 
There were significant differences between the levels of the factors "Perspective" $[F(1,16)=12.195, p=0.003]$, and "Timing" $[F(1,16)=48.963, p<0.001$; Figure 3C $]$. The pairwise comparisons of the synchronous and asynchronous conditions were only significant when the $1 \mathrm{PP}$ was adopted $(p<0.01$; Figure 1C). Thus, the illusion could be induced without the help of HMDs, but only when the mannequin was viewed from the 1PP.

\section{DISCUSSION}

Here, we have investigated the role played by the visual perspective in the mechanism underlying the self-attribution of an entire artificial body. Our results demonstrated that the first person visual perspective is critical for triggering the illusion of full-body ownership. This is an important observation as it shows that the very basic sensation of owning one's body is the result of a constructive process where visual, tactile, and proprioceptive signals are integrated in ego-centric coordinates.

These findings are relevant for the formulation of general models of body ownership (Makin et al., 2008; Tsakiris, 2010; Ehrsson, 2011) and corroborate models that emphasize that multisensory integration in body-part-centered reference frames is a crucial mechanism for the self-attribution of limbs as well as entire bodies (Makin et al., 2008; Ehrsson, 2011). The results of the present study, together with those from a previous report from our lab (Petkova and Ehrsson, 2008), show that the illusion of perceiving an entire body as belonging to oneself depends on: (i) temporal congruency of the visual and tactile signals applied in peripersonal space (i.e., within reaching distance); (ii) the "new" body having a sufficiently humanoid body shape; and (iii) using the first person visual perspective. The same constraints apply to the experience of the rubber hand illusion, which is an experimental model to study the self-attribution of a single body limb (Botvinick and Cohen, 1998; Ehrsson et al., 2004; Tsakiris and Haggard, 2005; Costantini and Haggard, 2007; Lloyd, 2007; Tsakiris, 2010). Thus, it is likely that both phenomena rely on similar multisensory mechanisms. In this multisensory framework, the integration of temporally, and spatially congruent visual, tactile, and proprioceptive signals generates a perceptually coherent percept of one's own body (Makin et al., 2008; Petkova and Ehrsson, 2008; Ehrsson, 2011).

Our results help resolve a recent discussion in the literature about the role played by the visual perspective in full-body illusions (Ehrsson, 2007; Lenggenhager et al., 2007; Meyer et al., 2008). It also corroborates the interpretation that the present illusion is a genuine body ownership illusion and not merely a more general form of self-recognition, as is the case when recognizing oneself in a mirror or on a CCTV screen. However, the question of how one can accommodate the conceptual differences between the results presented here and the findings reported by other groups (Lenggenhager et al., 2007); see also the set-up used in (Tsakiris, 2008) and (Sforza et al., 2010) which utilize the 3PP to trigger a body ownership illusion remains to be determined. For example, in the "enfacement effect" (Tsakiris, 2008; Sforza et al., 2010), people show a self-bias on visual face recognition tasks after a period of looking at the face of another person sitting opposite themselves while synchronous touches are applied to both this face and their own. However, in these experiments the face of the other person was viewed from a 3PP, which is consistent with the fact that this effect was not associated with a consciously experienced body (face) illusion, as evident from the rather low questionnaire rating scores when potential illusory experiences were probed (Sforza et al., 2010).

Another study employed a similar set-up to induce an out-ofbody illusion (Lenggenhager et al., 2007). In this experiment, the participants were looking either at their own body or the body of a mannequin, dressed in the same way as the participant, projected $2 \mathrm{~m}$ in front of them via the video-technology. According to the questionnaire data, the participants reported a self-attribution of the seen (own vs. mannequin's) body only when the visuo-tactile stimulation was synchronized. However, it cannot be excluded that these affirmative ratings were simply the result of visual self-recognition processes in which the participant understood the spatial cognitive transformation of the set-up (i.e., that he/she was cognizant of being filmed from behind and of this image being projected in front of him/her). Thus, it is plausible that the participants in this experiment recognized the virtual body they saw as a visual representation of their own body, without necessarily experiencing a somatic illusion of ownership in the same way as in the rubber hand illusion or in the body-swap illusion described here and in Petkova and Ehrsson (2008). The key difference is that the illusion presented here involves a complete perceptual binding of visual, tactile, and proprioceptive information onto the mannequin's body, which is experienced, somatically, to be one's own body. According to the results presented here, when observing a mannequin in far extrapersonal space from a 3PP, as in the study by Lenggenhager et al. (2007), a somatic illusion of this kind is absent, or at least significantly weaker. In the first two experiments, reported here, where the participants were wearing the HMDs, we observed weak affirmative rating scores for the statement that the mannequin body was one's own also in the third person visual perspective condition with synchronous visuo-tactile stimulation (Figure 3A). However, the ratings scores were significantly lower in comparison to the condition where the 1PP was adopted and there were no significant changes in the threat-evoked SCRs (see Figures 3B,C). Thus, we interpreted this effect as a form of general self-recognition rather than a genuine body illusion. This conclusion would be consistent with our experience from pilot experiments (see also Ehrsson and Petkova's response to Meyer et al., 2008) that this "self-recognition" effect completely disappears if one tries to induce the illusion from a 3PP without HMDs. This is in stark contrast to the body-swap illusion, which can be elicited without the use of video-technology, as we have demonstrated here.

A careful reader might have noted that the $3 \mathrm{PP}$ condition in the experiments reported here differed from the 1PP condition both in terms of perspective as such, and in that the mannequin was observed in far extrapersonal space rather than in peripersonal space. Thus, in principle, both these factors could have contributed to the abolishment of the illusion observed in the 3PP conditions. When designing the experiments we found it very unlikely that the significant reduction in the strength of the illusion in the 3PP condition could simply be due to the fact the mannequin was positioned in the far extrapersonal space. Research in the rubber hand illusion has shown that rotations of the model hand so that it is no longer matches the posture of the hidden real hand eliminates the illusion even when the rubber hand is positioned in the peripersonal space (Pavani et al., 2000; Ehrsson et al., 2005; Tsakiris and Haggard, 2005; 
Costantini and Haggard, 2007). In addition, it has been shown that the rubber hand illusion breaks down if the rubber hand is placed in far extrapersonal space (Lloyd, 2007). Thus, the most likely explanation is that both the $1 \mathrm{PP}$ and the presentation of the body in peripersonal space are necessary conditions to elicit a vivid full-body illusion. Future experiments could test this by presenting the mannequin close to the participant facing him or her within reaching distance (in peripersonal space).

The full-body illusion presented here is compatible with the neurophysiological and neuroimaging literature on multisensory integration of sensory information from the body. In the primate brain, regions in the intraparietal cortex, the inferior parietal cortex, the premotor cortex, and the putamen are implicated in integrating visual, tactile, and proprioceptive information in body-centered reference frames (Fogassi et al., 1992; Graziano and Gross, 1993; Graziano et al., 1997; Graziano, 1999; Graziano and Botvinick, 2002). These areas are active during visuo-tactile stimulation of the human body and show activity associated with the rubber illusion (Ehrsson et al., 2004, 2005) and the present full-body illusion according to very recent data from our lab (Petkova et al., submitted). Thus, the perceptual principles of the full-body illusion might derive from the multisensory integration mechanisms in operation in these areas (Ehrsson, 2010; Petkova et al., submitted).

In summary, the present experiments have revealed that the egocentric reference frame is important for the emergence of the corporeal sense of self. This finding represents a significant advance

\section{REFERENCES}

Aspell, J.E., Lenggenhager, B., and Blanke, O. (2009). Keeping in touch with one's self: multisensory mechanisms of selfconsciousness. PLoS ONE 4, e6488. doi: 10.1371/journal.pone.0006488

Blanke, O., Landis, T., Spinelli, L., and Seeck, M. (2004). Out-of-body experience and autoscopy of neurological origin. Brain 127, 243-258.

Blanke, O., and Metzinger, T. (2009). Full-body illusions and minimal phenomenal selfhood. Trends Cogn. Sci. 13, 7-13.

Blanke, O., and Mohr, C. (2005). Out-ofbody experience, heautoscopy, and autoscopic hallucination of neurological origin: implications for neurocognitive mechanisms of corporeal awareness and self-consciousness. Brain Res. Rev. 50, 184-199.

Blanke, O., Ortigue, S., Landis, T., and Seeck, M. (2002). Neuropsychology: stimulating illusory own-body perceptions. Nature 419, 269-270.

Botvinick, M., and Cohen, J. (1998). Rubber hands "feel" touch that eyes see. Nature 391, 756-756.

Burgess, N. (2006). Spatial memory: how egocentric and allocentric combine. Trends Cogn. Sci. 10, 551-557.

Costantini, M., and Haggard, P. (2007). The rubber hand illusion: sensitivity and reference frame for body ownership. Conscious. Cogn. 16, 229-240.
Ehrsson, H. H. (2007). The experimental induction of out-of-body experiences.

Ehrsson, H. H. (2011). "The concept of body ownership and its relationship to multisensory integration," in The Hand Book of Multisensory Processes, ed. B. Stein (Boston: MIT Press).

Ehrsson, H. H., Holmes, N. P., and Passingham, R. E. (2005). Touching a rubber hand: feeling of body ownership is associated with activity in multisensory brain areas. J. Neurosci. 25, 10564-10573.

Ehrsson, H. H., Rosen, B., Stockselius, A., Ragno, C., Kohler, P., and Lundborg, G. (2008). Upper limb amputees can be induced to experience a rubber hand as their own. Brain 131, 3443-3452.

Ehrsson, H. H., Spence, C., and Passingham, R. E. (2004). That's my hand! Activity in premotor cortex reflects feeling of ownership of a limb. Science 305, 875-877.

Fogassi, L., Gallese, V., di Pellegrino, G., Fadiga, L., Gentilucci, M., Luppino, G., Matelli, M., Pedotti, A., and Rizzolatti, G. (1992). Space coding by premotor cortex. Exp. Brain Res. 89, 686-690.

Gallagher,S. (2000). Philosophical conceptions of the self: implications for cognitive science. Trends Cogn. Sci. 4, 14-21.

Graziano, M. (1999). Where is my arm? The relative role of vision and proprioception in the neuronal repreScience 317, 1048.

in our understanding of the perceptual processes that mediate the feeling of owning a body. It also has important bearings on virtual reality research and advanced prosthetics, where one important goal is to develop humanoid robotic prostheses or simulated bodies that feel more like real ones (Rosén et al., 2009; Slater et al., 2009). By employing the principles of full-body ownership described here, one can optimize the projection of ownership onto simulated and artificial bodies in a wide range of industrial and clinical applications in these research fields (Ehrsson et al., 2008; Slater et al., 2008, 2009; Rosén et al., 2009).

\section{AUTHOR CONTRIBUTIONS}

Valeria I. Petkova and H. Henrik Ehrsson designed all experiments, Mehrnoush Khoshnevis conducted all three experiments, Valeria I. Petkova and Mehrnoush Khoshnevis analyzed the data, Valeria I. Petkova and H. Henrik Ehrsson wrote the manuscript.

\section{ACKNOWLEDGMENTS}

This work was funded by the European Research Council, the Human Frontier Science Programme, the Swedish Foundation for Strategic Research, and PRESENCCIA (Presence: Research Encompassing Sensory Enhancement, Neuroscience, CerebralComputer Interfaces, and Applications) project, a European Union-funded project under the Information Society Technologies Program. Valeria I. Petkova is supported by the graduate school of the Stockholm Brain Institute.

sentation of limb position. Proc. Natl. Acad. Sci. U.S.A. 96, 10418-10421.

Graziano, M., and Botvinick, M. (2002). "How the brain represents the body: insights from neurophysiology and psychology," in Common Mechanisms in Perception and Action: Attention and Performance XIX, eds W. Prinz and B. Hommel (Oxford: Oxford University Press), 136-157.

Graziano, M., and Gross, C. G. (1993). A bimodal map of space: somatosensory receptive fields in the macaque putamen with corresponding visual receptive fields. Exp. Brain Res. 97, 96-109.

Graziano, M., and Gross, C. G. (1998). Visual responses with and without fixation: neurons in premotor cortex encode spatial locations independently of eye position. Exp. Brain Res. 118, 373-380.

Graziano, M., Hu, X. T., and Gross, C. G. (1997). Visuospatial properties of ventral premotor cortex. J. Neurophysiol. 77, 2268-2292.

James, W. (1890). The Principles of Psychology. Available at: http:// psychclassics.yorku.ca/James/ Principles/

Jeannerod, M. (2003). The mechanism of self-recognition in humans. Behav. Brain Res. 142, 1-15.

Klatzky, R. L. (1998). "Allocentric and egocentric spatial representations: definitions, distinctions, and interconnections," in Spatial Cognition - An Interdisciplinary Approach to Representation and Processing of Spatial Knowledge (Lecture Notes in Artificial Intelligence 1404), eds C. Freksa, C. Habel, and K. F. Wender (Berlin: Springer), 1-17.

Lenggenhager, B., Mouthon, M., and Blanke, O. (2009). Spatial aspects of bodily self-consciousness. Conscious. Cogn. 18, 110-117.

Lenggenhager, B., Tadi, T., Metzinger, T., and Blanke, O. (2007). Video ergo sum: manipulating bodily selfconsciousness. Science 317, 1096-1099.

Lloyd, D. M. (2007). Spatial limits on referred touch to an alien limb may reflect boundaries of visuo-tactile peripersonal space surrounding the hand. Brain Cogn. 64, 104-109.

Maguire, E. A., Burgess, N., Donnett, J. G., Frackowiak, R. S., Frith, C. D., and O’Keefe, J. (1998). Knowing where and getting there: a human navigation network. Science 280, 921-924.

Makin, T. R., Holmes, N. P., and Ehrsson, H. H. (2008). On the other hand: dummy hands and peripersonal space. Behav. Brain Res. 191, 1-10.

Merleau-Ponty,M.(2005). Phenomenology of Perception. London: Routledge Classics.

Metzinger, T. (2003). Being No One: The Self-Model Theory of Subjectivity. Cambridge, MA: MIT Press. 
Meyer, K., Blanke, O., Ehrsson, H. H., and Petkova, V. (2008). Commentary E-letter.Science.Available at:http://www. sciencemag.org/content/317/5841/1048. abstract

Pavani, F., Spence, C., and Driver, J. (2000). Visual capture of touch: out-of-thebody experiences with rubber gloves. Psychol. Sci. 11, 353-359.

Petkova, V. I., and Ehrsson, H. H. (2008). If I were you: perceptual illusion of body swapping. PLoS ONE 3, e3832. doi: 10.1371/journal.pone.0003832

Petkova, V. I., and Ehrsson, H. H. (2010). Body Self-Perception. McGraw-Hill Yearbook of Science and Technology. New York: McGraw-Hill Companies, Inc., 50-53.

Rosén, B., Ehrsson, H. H., Antfolk, C., Cipriani, C., Sebelius, F., and Lundborg, G. (2009). Referral of sensation to an advanced humanoid robotic hand prosthesis. Scand. J. Plast. Reconstr. Surg. Hand Surg. 43, 260-266.

Sforza, A., Bufalari, I., Haggard, P., and Aglioti, S.M. (2010). My face in yours: visuo-tactile facial stimulation influences sense of identity. Soc. Neurosci. 5, 148-162.

Slater, M., Perez-Marcos, D., Ehrsson, H. H., and Sanchez-Vives, M. V. (2008). Towards a digital body: the virtual arm illusion. Front. Hum. Neurosci. 2:6. doi: 10.3389/neuro.09.006.2008

Slater, M., Perez-Marcos, D., Ehrsson, H. H., and Sanchez-Vives, M. V. (2009). Inducing illusory ownership of a virtual body. Front. Neurosci. 3:2. doi: 10.3389/neuro.01.029.2009

Slater, M., Spanlang, B., Sanchez-Vives, M. V., and Blanke, O. (2010). First person experience of body transfer in virtual reality. PLOS ONE
5, e10564. doi: 10.1371/journal. pone.0010564

Tsakiris, M. (2008). Looking for myself: current multisensory input alters self-face recognition. PLOS ONE 3, e4040. doi: 10.1371/journal.pone.0004040

Tsakiris, M. (2010). My body in the brain: a neurocognitive model of body-ownership. Neuropsychologia 48, 703-712.

Tsakiris, M., and Haggard, P. (2005). The rubber hand illusion revisited: visuotactile integration and self-attribution. J. Exp. Psychol. Hum. Percept. Perform. 31, 80-91.

Vogeley, K., and Fink, G. R. (2003). Neural correlates of the first-person-perspective. Trends Cogn. Sci. 7, 38-42.

Conflict of Interest Statement: The authors declare that the research was conducted in the absence of any commercial or financial relationships that could be construed as a potential conflict of interest.

Received: 19 November 2010; accepted: 21 February 2011; published online: 07 March 2011.

Citation: Petkova VI, Khoshnevis $M$ and Ehrsson HH (2011) The perspective matters! Multisensory integration in ego-centric reference frames determines full-body ownership. Front. Psychology 2:35. doi: 10.3389/ fpsyg.2011.00035

This article was submitted to Frontiers in Cognition, a specialty of Frontiers in Psychology.

Copyright $(2011$ Petkova, Khoshnevis and Ehrsson. This is an open-access article subject to an exclusive license agreement between the authors and Frontiers Media SA, which permits unrestricted use, distribution, and reproduction in any medium, provided the original authors and source are credited. 